\title{
Cisplatin, VP-16-213 and MGBG (Methylglyoxal bis guanylhydrazone) combination chemotherapy in refractory lymphoma, a phase II study
}

\author{
Lyubica Dabich and Marcia K. Liepman \\ Division of Hematology/Oncology, Department of Internal Medicine, University of Michigan Medical \\ School, Ann Arbor, Michigan, USA
}

Key words: refractory non-Hodgkin's lymphoma, cisplatin, VP-16-213 and MGBG

\section{Summary}

In an effort to improve the treatment of patients with refractory or recurrent lymphoma, we developed a protocol using cis-platinum combined with two other agents of known efficacy in these disorders but with differing side effects: VP-16 and MGBG. Twenty-six eligible patients were treated with this regimen. There were 15 men and 11 women with a median age of 54 years (22-73), and performance status of $1(0-3)$. Their diagnoses were Hodgkin's disease 5 and non-Hodgkin's lymphoma [NHL] 21 which included 11 with diffuse histocytic lymphoma [DHL]. The median number of chemotherapy regimens was $2(1-5) ; 12$ also received radiotherapy. Twenty patients are evaluable for response: $15 \mathrm{NHL}$ and 5 Hodgkin's disease. Three patients, all of whom had DHL entered complete remission (20\%) with a median time to treatment failure of $71 / 2$ months. Six NHL (40\%) and one Hodgkin's disease $(20 \%)$ patients entered a partial remission. There were three early deaths: one due to progressive disease, one to acute respiratory failure, and one with disease status undocumented. Toxicity included leukopenia, thrombocytopenia, anorexia, nausea, vomiting, stomatitis, alopecia, renal failure, profound peripheral neuropathy, and hypersensitivity vasculitis. Treatment was stopped because of the latter two. These agents are non-crossresistant with doxorubicin-containing regimens. The drugs are possibly synergistic and modestly active with moderate to severe toxicity.

\section{Introduction}

The poor response rate with salvage chemotherapy for patients with lymphoma who have either failed to enter into remission or who have relapsed from their initial combination chemotherapy prompted interest in a new type of drug: cis-diamminedichloroplatinum [1-2]. Following the reports of its efficacy in these patients as a single agent, it was tested in 2 and 3 drug combinations [3-16]. These are summarized in Table 1 . We have recently described the results of such a three drug combination. Cisplatin, vinblastine and bleomycin, given to 17 very heavily pretreated patients, yielded responses in 9 ( 3 complete and 6 partial) [14]. Toxicity included severe myelosuppression and mild decrease in creatinine clearance in patients receiving 2 or more courses. There were no drug related deaths. Most responders achieved partial remission after the first course. Furthermore, many of the nonresponders demonstrated a rapid tumor shrinkage but equally rapid regrowth and thus could not be called responders. These results prompted us to consider another combination with cisplatinum, and we selected VP-16 and MGBG. VP-16 is a synthetic congener of podophyllotoxin, the crystalline extract of the plant podophyllum peltatum. It was shown to have a response rate of $14 \%$ in 64 patients with Hodgkin's and $23-32 \%$ in over 100 patients with non-Hodgkin's lymphoma [17]. Its major tox- 
Table I. Literature survey

\begin{tabular}{|c|c|c|c|c|c|c|c|c|c|c|c|c|c|c|c|c|c|c|c|c|c|c|}
\hline \multirow[b]{2}{*}{ Study } & \multirow[b]{2}{*}{ Ref } & \multirow{2}{*}{\multicolumn{2}{|c|}{ Drugs }} & \multicolumn{7}{|c|}{ Non-Hodgkin's } & \multicolumn{6}{|c|}{ Hodgkin's disease } & \multicolumn{6}{|c|}{ Unknown distribution } \\
\hline & & & & Total & Eval & $\mathrm{CR}$ & $\%$ & PR & $\%$ & $\begin{array}{c}\text { \% Total } \\
\text { resp }\end{array}$ & Eval & $\mathrm{CR}$ & $\%$ & PR & $\%$ & $\begin{array}{c}\% \text { Total } \\
\text { resp }\end{array}$ & Eval & $\mathrm{CR}$ & $\%$ & PR & $\%$ & $\begin{array}{c}\% \text { Total } \\
\text { resp }\end{array}$ \\
\hline Rossof & 1 & PT & & 25 & 17 & & & 1 & 6 & 6 & 8 & 1 & 13 & 1 & 13 & 26 & & & & & & \\
\hline Cavalli & 2 & PT & & 31 & 19 & & & 5 & 26 & 26 & 8 & 1 & 13 & & & 13 & & & & & & \\
\hline Bender & 21 & VP-16 & & 20 & 19 & 1 & 5 & 7 & 37 & 42 & & & & & & & & & & & & \\
\hline Kroner & 5 & VP-16 & & 20 & 13 & & & 5 & 38 & 38 & 7 & & & 5 & 71 & 71 & & & & & & \\
\hline Knight & 22 & & MGBG & 54 & 44 & 1 & 2 & 8 & 18 & 20 & 10 & & & 3 & 30 & 30 & & & & & & \\
\hline Warrell & 23 & & MGBG & 46 & 27 & & & 10 & 37 & 37 & 13 & & & 6 & 46 & 46 & & & & & & \\
\hline Judson & 3 & PT VP-16 & & 25 & 17 & 5 & 29 & 4 & 24 & 53 & & & & & & & & & & & & \\
\hline Kaplan & 4 & PT VP-16 & & 58 & 53 & 6 & 11 & 11 & 21 & 32 & & & & & & & & & & & & \\
\hline Kroner & 5 & PT VP-16 & & 22 & 18 & & & 5 & 38 & 38 & 7 & & & 5 & 71 & 71 & & & & & & \\
\hline Von Heyden & 6 & PT VP-16 & & 15 & 4 & & & 1 & 25 & 25 & 11 & & & 2 & 18 & 18 & & & & & & \\
\hline Fosser & 7 & PT VP-16 & 6 PRED & 18 & 6 & & & 2 & 33 & 33 & 12 & 3 & 25 & 2 & 17 & 41 & & & & & & \\
\hline Dabich & & PT VP-16 & 6 MGBG & 27 & 15 & 3 & 20 & 6 & 40 & 60 & 5 & & & 1 & 20 & 20 & & & & & & \\
\hline Williams & 8 & PT VP-16 & $\begin{array}{l}6 \mathrm{CCNU} \\
\mathrm{BCNU} \\
\text { MTX-L }\end{array}$ & 25 & 18 & & & 3 & 17 & 17 & 7 & 1 & 14 & & & 14 & & & & & & \\
\hline Silverman & 9 & PT VP-16 & 6 ARA-C & 16 & & & & & & & & & & & & & 15 & 1 & 7 & 9 & 60 & 67 \\
\hline Dana & 10 & PT AMSA & A MGBG & 37 & 30 & & & 13 & 44 & 44 & & & & & & & & & & & & \\
\hline Camacho & 11 & PT VDS & MGBG & 16 & & & & & & & & & & & & & 14 & 2 & 14 & 6 & 43 & 57 \\
\hline Tseng & 12 & PT VM-26 & $6 \mathrm{ARA}-\mathrm{C}$ & 21 & 21 & 2 & 10 & 7 & 33 & 43 & & & & & & & & & & & & \\
\hline Spiers & 13 & PT VM-26 & $\begin{array}{l}6 \mathrm{BCNU} \\
\mathrm{BL} \\
\mathrm{DEX}\end{array}$ & 20 & & & & & & & & & & & & & 13 & 2 & 15 & 5 & 38 & 53 \\
\hline Liepman & 14 & PT VLB B & & 17 & 14 & 3 & 21 & 2 & 14 & 35 & 3 & & & 3 & 100 & 100 & & & & & & \\
\hline Corder & 15 & PT VLB B & & 13 & 13 & & & 5 & 38 & 38 & & & & & & & & & & & & \\
\hline Velasquez & 16 & PT ARA-C & $C D E X$ & 48 & 43 & 11 & 26 & 11 & 26 & 52 & & & & & & & & & & & & \\
\hline
\end{tabular}

Drugs: $\mathrm{BCNU}=$ Carmustine, $\mathrm{CCNU}=$ Lomustine, $\mathrm{BL}-\mathrm{Bleomycin}$ MTX-L $=$ Methotrexate with leucovorin rescue $\mathrm{VDS}=$ Vindesine, $\mathrm{VLB}=$ Velban, $\mathrm{VM}-26=$ Teniposide $\mathrm{PRED}=$ Prednisone, $\mathrm{DEX}=$ Dexamethasone AMSA $=$ Amsacrine, ARA-C $=$ Cytosine arabinoside

icity is reversible myelosuppression, particularly leukopenia and anemia [17-19]. Methylglyoxal bis guanylhydrazone is a drug in which there is renewed interest. It had been used previously as methy-gag [20-23]. Warrell noted partial responses in 6 of 13 evaluable patients with refractory Hodgkin's disease and 10 of 27 patients with non-Hodgkin's lymphomas [23]. Toxicity was mild and consisted mainly of muscular weakness, myalgia, mucositis, and diarrhea. Myelosuppression was minimal which made this agent attractive for use in combination with myelotoxic drugs.

From January, 1981 until January, 1985 this pro- tocol was used to treat patients with refractory lymphoma, both Hodgkin's and non-Hodgkin's type. We are reporting our results and toxicity in 27 of these patients.

\section{Treatment}

The protocol is outlined in Table 2, which also illustrates the adjustment for hematologic nadir and renal impairment. The courses were repeated every 28 days. 
Table 2. Protocol

\begin{tabular}{|c|c|c|c|c|}
\hline & Drugs & Dose & Days of cycle & \\
\hline & $\begin{array}{l}\text { Cisplatin } \\
\text { VP-16 } \\
\text { MGBG }\end{array}$ & $\begin{array}{l}60 \mathrm{mg} / \mathrm{M}^{2} \\
80 \mathrm{mg} / \mathrm{M}^{2} \\
500 \mathrm{mg} / \mathrm{M}^{2}\end{array}$ & $\begin{array}{l}1 \\
1,2,3 \\
1,8,15\end{array}$ & \\
\hline \multicolumn{5}{|c|}{ Modifications: hematologic nadirs } \\
\hline PMNS & Platelets & $\mathrm{CISPT} / \mathrm{M}^{2}$ & $\mathrm{VP}-16 / \mathrm{M} / \mathrm{M}^{2}$ & $\mathrm{MGBG}^{2}$ \\
\hline $\begin{array}{l}>1500 / \mathrm{mm}^{3} \\
500-999 / \mathrm{mm}^{3} \\
<499\end{array}$ & $\begin{array}{l}>100,000 / \mathrm{mm}^{3} \\
50-74,999 / \mathrm{mm}^{3} \\
<49,999\end{array}$ & $\begin{array}{l}\text { Same } \\
\text { Same } \\
60 \mathrm{mg}\end{array}$ & $\begin{array}{l}\text { Increase to } 100 \mathrm{mg} \\
60 \mathrm{mg} \\
40 \mathrm{mg}\end{array}$ & $\begin{array}{l}\text { Same } \\
\text { Same } \\
250 \mathrm{mg}\end{array}$ \\
\hline \multicolumn{5}{|l|}{ Renal } \\
\hline \multicolumn{5}{|c|}{$\begin{array}{l}\text { Rise in maximum serum creatinine } \\
\text { above baseline (mg \%) }\end{array}$} \\
\hline & & $\mathrm{CISPT} / \mathrm{M}^{2}$ & & $\mathrm{MGBG} / \mathrm{M}^{2}$ \\
\hline & $\begin{array}{l}<.5 \\
>.5 \text { but }<.75 \\
>.75 \text { but }<1.0 \\
>1.0\end{array}$ & $\begin{array}{l}\text { Same } \\
\text { Decrease } 25 \% \\
\text { Decrease } 50 \% \\
\text { Hold }\end{array}$ & & $\begin{array}{l}\text { Same } \\
250 \mathrm{mg} \\
250 \mathrm{mg} \\
\text { Hold }\end{array}$ \\
\hline
\end{tabular}

\section{Material and methods}

\section{Eligibility criteria}

All patients with histologically confirmed refractory Hodgkin's or non-Hodgkin's lymphoma who had measurable disease and performance status equal to 3 or better by SWOG group criteria were eligible for entry onto this protocol. Patients with Hodgkin's disease should have received a doxorubicin containing regimen prior to entry onto this study. There were no age restrictions and no limitation on the number of previous regimens. The initial WBC count was to be equal to or greater than $3000 / \mathrm{cu} \mathrm{mm}$ and the platelet count equal to or greater than $75,000 / \mathrm{cu} \mathrm{mm}$. The serum creatinine could be no greater than $1.5 \mathrm{mg} \%$. The creatinine clearance following hydration was to be greater than $50 \mathrm{cc} /$ minute. Patients could have no evidence of active central nervous system involvement. If such disease were present, specific treatment aimed at CNS lymphoma was to be underway before the patient could begin chemotherapy with this pro- gram. Patients were to be 3 weeks past previous chemotherapy or radiation therapy at the onset. The period was 4 weeks for patients who had received nitrosoureas. It was necessary for patients to give informed consent in keeping with our institutional policies and a signed statement to that effect was to be a part of the record. We planned to give patients allopurinol unless they were allergic to this medication.

\section{Pretreatment requirements}

Pretreatment evaluation included history and physical examination with documentation of symptoms, disease activity, tumor measurements, and performance status. Laboratory parameters included CBC with differential and platelet count, uric acid, electrolytes, magnesium, and liver function studies (SGOT, SGPT, LDH, alkaline phosphatase and bilirubin). To establish the renal function we obtained BUN and serum creatinine, urinalysis with microscopic examination of the sediment and a 24 hour urine for creatinine and protein. IVP or renal 
ultrasound was used to rule out hydronephrosis. Other investigations included chest $\mathrm{x}$-ray and electrocardiogram.

\section{Criteria for response}

Complete remission (CR) was defined as the disappearance of all clinical evidence of tumor on physical examination, $\mathrm{x}$-ray or biochemical evaluation for more than 1 month. Partial remission (PR) was $50 \%$ or greater decrease by physical examination, roentgenogram and/or CT scan in the sum of the products of the perpendicular diameters of all measured lesions lasting more than 1 month. There could be no simultaneous increase in the size of any lesion or appearance of any new lesions. A partial remission in metastatic liver disease consisted of a $50 \%$ reduction in the summation of all liver measurements below the costal margin and improvement of abnormal liver function tests to normal. Minor response was a $25 \%$ decrease in all measurable disease but less than that required for a partial remission. Stabilization was a less than $25 \%$ decrease or increase in measurable disease for more than 2 months. For progression (Progr) there was to be the appearance of either new lesions or a greater than $25 \%$ increase in measurable disease. The subjective response was measured by the SWOG performance scale. The duration of response was measured from the initiation of the study until a $25 \%$ increase in the sum of all tumor measurements was reached. An adequate trial was defined as one cycle of therapy with objective disease progression or 2 cycles of therapy.

\section{Results}

The individual patients are described in Table 3 and toxicity in Table 4. Although 27 patients were entered onto this protocol, one patient with Hodgkin's disease was ineligible because he had not received a doxorubicin containing regimen prior to this therapy. The 26 eligible patients included 15 men and 11 women with a median age of 54 years, range 22 to 73 , and a performance status of 1 , range
0 to 3 . There were 5 with Hodgkin's disease, and 21 with non-Hodgkin's lymphoma, sub-divided into 11 diffuse histiocytic lymphoma (DHL), 3 diffuse mixed lymphoma (DML), 3 diffuse poorly differentiated lymphoma (DPDLL), 2 nodular poorly differentiated lymphocytic lymphoma (NPDLL) and 2 well differentiated lymphocytic lymphoma (WDLL). Bone marrow involvement was present in 6 and bone involvement in 5 patients. These patients were heavily pretreated. The median number of previous chemotherapy regimens was 2 with a range of $1-5$. Twelve of the 26 patients had also received radiotherapy at some time. Two patients were treated with less than 1 course, 10 patients 1 course, seven 2 , two 3 , three 5 , two 6 and one 8 courses of cisplatin, VP-16, and MGBG. Patients in $\mathrm{PR}$ continued to be treated as long as they responded. Twenty of the twenty six eligible patients, 15 with NHL and 5 with Hodgkin's disease, were evaluable for response. There were three complete remissions in patients with DHL [3/15 NHL $=20 \%$ ] with time to treatment failure $5,7 \frac{1}{2}$, and 42 months. There were 3 early deaths, one due to disease progression prior to completion of the first course, and one immediately after. Unfortunately the patient was transferred to another hospital and the measurable disease was not documented. The third patient died of acute respiratory failure, the question of its relationship to chemotherapy is not resolved. Two patients had therapy discontinued because of profound peripheral neuropathy and hypersensitivity vasculitis, and one refused to complete the first course.

Treatment in 67 courses resulted in a median white count of $1800 / \mathrm{cu} \mathrm{mm}$, range $100-14,200$, platelets $93,000 / \mathrm{cu} \mathrm{mm}$, range 3200-391,000. The doses were sufficiently high that only 3 patients received the prescribed chemotherapy. Although MGBG should have been given, the usual adjustment for myelosuppression was the withholding of Day 15 and/or Day 8 MGBG. This was done even on the first course. There was severe drug induced renal failure in one patient and modest renal impairment in two others. In one of these there was a response to modulation of dose. Infections occurred in 9 of 67 courses and bleeding in 3. Severe nausea and vomiting was noted at some time in one- 
Table 3. Individual patient characteristics

\begin{tabular}{|c|c|c|c|c|c|c|c|c|c|c|c|c|c|}
\hline \# & Diag & Age & Sex & Race & Previous chemotherapy & $\operatorname{Rad}$ & P.S. & B.M. & Bone & Sites of disease & $\begin{array}{l}\text { No. of } \\
\text { courses }\end{array}$ & Outcome & $\begin{array}{l}\text { Duration } \\
\text { months }\end{array}$ \\
\hline 2 & Hodgkins & 22 & $\mathrm{~F}$ & W & $\begin{array}{l}\text { MOPP, VELB, COP, } \\
\text { CHLOP, C-MOPP }\end{array}$ & No & 1 & & & Lung, Nodes & 1 & PROGR & \\
\hline 3 & & 26 & F & W & MOPP, CHOP, PVB & Yes & 1 & & + & Pancreas, Nodes, Flank & 2 & PR & \\
\hline 5 & & 26 & M & W & MOPP, C-MOPP & No & 1 & & & Nodes, Kidney & 3 & INELIG & \\
\hline 11 & & 26 & $\mathrm{~F}$ & W & MOPP, ABVD & Yes & 0 & & & $\begin{array}{l}\text { Breast mass, Chest wall, } \\
\text { Mediastinum }\end{array}$ & 1 & PROGR & \\
\hline 18 & & 22 & M & W & MOP-BAP & No & 1 & & & $\begin{array}{l}\text { Adrenal, Mediastinum, } \\
\text { Ext Abd dis }\end{array}$ & 1 & PROGR & \\
\hline 23 & & 24 & $\mathrm{~F}$ & $w$ & MOPP, ABVD, BCVPP & Yes & 0 & & & Nodes & 1 & PROGR & \\
\hline 7 & WDLL & 58 & $\mathbf{M}$ & $\mathrm{N}$ & $\begin{array}{l}\mathrm{COP}, \mathrm{COP}, \mathrm{VCR} / \mathrm{CHL} \\
\mathrm{AOP}, \mathrm{POACH}\end{array}$ & No & 2 & + & + & Liver, Nodes & 2 & PROGR & \\
\hline 12 & & 54 & M & $\mathrm{w}$ & CHOP, COP, COAP, COMLA & No & 0 & + & & Nodes, Liver, ABD Mass & 1 & PR & \\
\hline 9 & NPDLL & 47 & F & W & $\begin{array}{l}\text { CHOP, X2, PVB, CCNU/ } \\
\text { PRO, CHL/PRED }\end{array}$ & No & 1 & & & ABD Mass, Nodes & 8 & PR & \\
\hline 16 & & 37 & M & $w$ & $\mathrm{CHOP}, \mathrm{PVB}, \mathrm{CHL} / \mathrm{CCNU}$ & No & 1 & + & & Nodes & 2 & PROGR & \\
\hline 17 & DPDLL & 51 & $\mathrm{~F}$ & W & CHOP & No & 0 & + & & Nodes & 3 & PR & \\
\hline 19 & & 68 & M & w & CHOP, COP & No & 2 & + & & Nodes, Liver, Lung & 1 & NE-RFT & \\
\hline 22 & & 61 & M & W & CHOP & Yes & 1 & & & Abd Mass & $<1$ & NE-TOX ${ }^{1}$ & \\
\hline 13 & DML & 66 & M & W & BACOP, CYTOXAN & No & 1 & & & Small bowel, Nodes & 6 & $\mathrm{PR}$ & \\
\hline 15 & & 60 & M & $w$ & COP, CHLOP, CHOP & Yes & 1 & + & & Lung, Pleura, Chest wall & 2 & $\mathrm{PR}$ & \\
\hline 21 & & 54 & $\mathrm{~F}$ & W & $\mathrm{CHOP}$ & No & 1 & & & Liver, Nodes, ABD Mass & 5 & $\mathrm{PR}$ & \\
\hline 1 & DHL & 52 & M & $\mathrm{B}$ & CHOP, COP, ARA-C-MTX & Yes & 0 & & & Lung, Nodes & 6 & $\mathrm{CR}$ & $71 / 2$ \\
\hline 4 & & 41 & $M$ & W & BACOP, ARA-C-MTX & Brain & 1 & & & Abd wall, CSF & 2 & PROGR & \\
\hline 6 & & 63 & F & $\mathrm{W}$ & CHOP, COP-BL & Yes & 1 & & & $A b d$ & 2 & PROGR & \\
\hline 8 & & 31 & M & W & CHOP, HIGH DOSE ADRIA & No & 1 & & + & Tonsils, Nodes, Liver & 2 & PROGR & \\
\hline 10 & & 70 & $\mathrm{~F}$ & W & CHOP & No & 1 & & & Nodes & 1 & NE-ED & \\
\hline 14 & & 54 & $\mathbf{M}$ & W & CHOP & Yes & 1 & & & Nodes, Skin, CNS, Abd mass & 5 & CR. & 5 \\
\hline 20 & & 49 & $\mathrm{~F}$ & W & HIGH DOSE ADRIA & Yes & 0 & & + & Nodes, Palate & 5 & $\mathrm{CR}$ & 42 \\
\hline 24 & & 43 & F & W & CHOP & Yes & 0 & & + & Nodes & 1 & PROGR & \\
\hline 25 & & 61 & M & w & CHOP, MOPP & Yes & 0 & & & Liver, Nodes & 1 & NE-TOX ${ }^{2}$ & \\
\hline 26 & & 73 & $\mathbf{M}$ & B & $\mathrm{CHOP}$ & No & 3 & & & Tonsils, Nodes & $<1$ & NE-ED & \\
\hline 27 & & 51 & M & w & BACOP & Yes & 1 & & & Abd mass, Nodes & 1 & NE-ED & \\
\hline \multicolumn{2}{|r|}{ Summary: } & \multicolumn{8}{|c|}{ CR-3, PR-7, PROGR-10, NE-ED-3, TOX-2, INELIG-1, RFT-1 } & $\begin{array}{l}{ }^{\text {ISevere neuropathy }} \\
{ }^{2} \text { Hypersensitivity vasculitis }\end{array}$ & & & \\
\hline
\end{tabular}

Drug combinations

ABVD: $\quad$ Adriamycin, Bleomycin, Vinblastine, DTIC

AOP: $\quad$ Adriamycn, Vincristine, Prednisone

ARA-C-MTX: Cytosine Arabinoside-Methotrexate

BACOP: Bleomycin, Adriamycin, Cyclophosphamide, Vincristine, Prednisone

BCVPP: BCNU, Cyclophosphamide, Vinblastine, Procarbazine, Prednisone

CCNU/PRO: CCNU/Procarbazine

CHL-O-P: Chlorambucil, Vincristine, Prednisone

CHL/PRED: Chlorambucil/Prednisone

CHOP: Cyclophosphamide, Adriamycin, Vincristine, Prednisone

C-MOPP: Cyclosphosphamide, Vincristine, Procarbazine, Prednisone

COAP: Cyclophosphamide, Vincristine, Cytosine Arabinoside, Prednisone
COMLA: Cyclophosphamide, Vincristine, Methotrexate, Levcovorin, Adriamycin

COP: $\quad$ Cyclophosphamide, Vincristine, Prednisone

COP-BL: Cyclophosphamide, Vincristine, Prednisone, Bleomycin

HIGH DOSE Adriamycin, Vincristine, Prednisone, Cytosine

ADRIA Arabinoside, Cyclophosphamide

MOP-BAP: Nitrogen Mustard, Vincristine, Procarbazine, Bleomycin, Adriamycin, Prednisone

MOPP: Nitrogen Mustard, Vincristine, Procarbazine, Prednisone

POACH: Prednisone, Vincristine, Cytosine Arabinoside, Cyclophosphamide, Adriamycin

PVB: $\quad$ Platinum, Vinblastine, Bleomycin

VELB Vinblastine

VCR/CHL: Vincristine/Chlorambucil 
Table 4. Toxicity

WBC median $1800 / \mathrm{cu} \mathrm{mm}(100-14,200)$

Platelets $93,000 / \mathrm{cu} \mathrm{mm}(3200-391,000)$

\begin{tabular}{lll}
$\geq 3 \mathrm{~N} / \mathrm{V}$ or & $>5$ emesis/day & $9 / 27$ patients \\
$\geq 3$ Anorexia or & $5-10 \% \mathrm{wt}$ loss & $1 / 27$ patients \\
$\geq 3$ Stomatitis or & $>3$ discrete ulcers & $2 / 27$ patients \\
Hepatic & & $0 / 27$ patients \\
$\geq 2$ Renal or & $\begin{array}{l}\text { Bun } 51-75 \text { or creatinine } \\
\end{array}$ & $3 / 27$ patients* \\
$\geq 3$ CNS or & $\begin{array}{l}2.6-3.0 \mathrm{mg} / \mathrm{dl} \text {. } \\
\text { severe paresthesias }\end{array}$ & $1 / 27$ patients \\
$\geq 3$ Skin or & $\begin{array}{l}\text { moist disquamation } \\
\text { with ulceration }\end{array}$ & $1 / 27$ patients \\
$\geq 2$ Alopecia or & pronounced hair loss & $1 / 27$ patients \\
Infections & $9 / 67$ courses & \\
Bleeding & $3 / 67$ courses & \\
\hline
\end{tabular}

* Responded to change in dose: $1 / 3$ patients

third of the patients, but severe diarrhea occurred in only 1 patient and stomatitis in 2 . As noted there was discontinuation of protocol due to severe peripheral neuropathy in one patient and to skin hypersensitivity in another. Alopecia was not a problem. Hypoglycemia was not seen.

\section{Discussion}

The addition of other agents to cis-platinum seems to confer some added efficacy, since results with cis-platinum as a single agent were modest with few complete responders (Table 1). Unfortunately, the small numbers of patients and the relatively large numbers of protocols with different doses and schedules preclude the use of statistical analysis to sort out the contribution of each agent. For example, Judson and Wiltshaw, [3] i.e., used a dose schedule of cis-platinum $50 \mathrm{mg} / \mathrm{m}^{2}$ i.v. daily with hydration plus VP-16 $100 \mathrm{mg} / \mathrm{m}^{2}$ i.v. days $1-3$, repeated every 3 weeks, whereas Kaplan et al. [4] administered cis-platinum $20 \mathrm{mg} / \mathrm{m}^{2}$ and VP-16 80 $\mathrm{mg} / \mathrm{m}^{2}$, both daily for 5 days every $3-4$ weeks. The Judson and Wiltshaw regimen produced the best complete response rate $(29 \%)$ in patients with non-Hodgkin's lymphoma, but it was only of 12 weeks duration. The combination of cis-platinum, cytosine arabinoside and decadron also resulted in a significant complete response $(26 \%)$ with a more durable remission. Like these latter investigators we achieved our best responses in patients with large cell lymphoma, all of whom had been treated initially with doxorubicin containing regimens, suggesting these drugs are non-cross resistant with the front line therapy. One of the responders had received multiple combinations [3] prior to this one. The remissions were fairly long-lived with a median time to treatment failure of $7 \frac{1}{2}(5-42)$ months, but the CR rate $(20 \%)$ is not as good than that of the cytosine arabinoside combination. Toxicity was moderately severe and, mainly, reversible. Renal impairment persisted in $2 / 3$ patients.

In summary, the combination of cis-platinum, VP-16 and MGBG constitutes a modestly active and moderately to severely toxic regimen for the management of patients with relapsed lymphoma. Its efficacy in this group of patients might warrant further exploration of these potentially synergistic drugs in protocols with refractory aggressive lymphomas.

\section{Acknowledgement}

Supported by a Grant from The National Cancer Institute NO1-CM-07405

\section{References}

1. Rossof AH, Coltman Jr CA, Jones SE, Talley RW: Phase II Evaluation of Cis-Dichlorodiammineplatinum (II) in Lymphomas: A Southwest Oncology Group Study. Cancer Treat Rep 63:1605-08, 1979

2. Cavalli F, Junghi WF, Nissen NI, Pajak TF, Coleman M, Holland JF: Phase II Trial of Cis-Dichlorodiammineplatinum (II) in Advanced Malignant Lymphoma. A Study of the Cancer and Acute Leukemia Group B. Cancer 48: 1927-30, 1981

3. Judson IR, Wiltshaw E: Cis-Dichlorodiammineplatinum (Cis-Platinum) and Etoposide (VP-16) in Malignant Lymphoma - an Effective Salvage Regimen. Cancer Chemother Pharmacol 14:258-61, 1981

4. Kaplan RS, Budman D, Gottlieb A, Anderson J: Phase II Trial of Etoposide (VP-16) and Cisplatin (DDP) in Refractory Non-Hodgkin's Lymphomas (NHL). A CALGB Study. Proc Am Soc Clin Oncol 4: 203, 1985 (Abstr)

5. Kroner T, Obrecht JP, Junghi WF: Etoposide as Single 
Agent and in Combination with Cis-Platinum for Malignant Lymphomas. Cancer Treat Rev 9 (Supple A) 39-43, 1982

6. Von Heyden HW, Scherpe A, Nagel GA: Cis-Dichlorodiammineplatinum (II) (Cis-Platinum) and Etoposide for Patients with Refractory Lymphomas. Cancer Treat Rev 9 (Supple A) 45-52, 1982

7. Fosser VP, Salvagno L, Segati R, Pappagallo GL, Ferrazzi E, Sileni VC, Fiorentino MV: Cis-Dichlorodiammineplatinum (II), VP16-213 and Prednisone (DVP Regimen) in the Treatment of Pretreated Advanced Malignant Lymphomas. Tumori 68:515-8, 1982

8. Williams SF, Ultman JE, Gaynor E, Watson S: Management of Advanced, Resistant Hodgkin's Disease and NonHodgkin's Lymphoma: A Phase II Study. Cancer Treat Rep 70:1033-4, 1986

9. Silverman L, Jones R, Norton L, Cuttner J, Malamud S, Holland JF: Combination Chemotherapy for Refractory Lymphoma with Cytosine Arabinoside (A) Cisplatin (P) and Etoposide (E): APE. Proc Am Soc Clin Oncol 3:246, 1984 (Abstr)

10. Dana BW, Jones SE, Coltman C, Stuckey WJ: Salvage Treatment of Unfavorable Non-Hodgkin's Lymphoma with Cisplatin, Amsacrine, and Mitoguazone: A Southwest Group Pilot Study. Cancer Treat Rep 70:291-2, 1986

11. Camacho F, Oken M, O'Connell M, Perri R, Phillips V, Wiernik P: Salvage Therapy in Patients (Pts) with Refractory Malignant Lymphoma Using Methylglyoxal Bisguanylhydrazone (MGBG), Vindesine (VDS) and Cisplatinum (CPPD). Proc Am Soc Clin Oncol 3:242, 1984 (Abstr)

12. Tseng Jr A, Jacobs C, Coleman C, Horning S, Lewis B, Rosenberg S: Treatment of Refractory Non-Hodgkin's Lymphomas of Unfavorable Histology with Tenoposide (VM-26), Cytosine Arabinoside (Ara-C) and Cisplatin. Proc Am Soc Clin Oncol 4:203, 1985 (Abstr)

13. Spiers ASD, Weens JH, Parkinson DR, O'Connell MJ: Treatment of Refractory Lymphomas with BCNU, Bleomycin, VM-26, Dexamethasone and Cisplatin (BBVDD Regimen). Proc Am Soc Clin Oncol 3:237, 1984 (Abstr)

14. Liepman MK, Wheeler RH, Zuckerman KS, LoBuglio AF: Combination Chemotherapy of Refractory Lymphoma with Cis-Dichlorodiammineplatinum, Vinblastine and Bleomycin. Cancer 50:2736-39, 1982

15. Corder MP, Clamon GH: Salvage Therapy of Aggressive Non-Hodgkin's Lymphoma with a Combination of Vinblastine, Bleomycin and Cisplatin. Cancer 54:202-6, 1984

16. Velasquez W, Caranillas F, McLaughlin P, Plunkett W, Hagemeister F, Swan F, Freireich EJ: Combination of Cisplatin-Ara-C and Decadron in Relapsing Lymphoma. Proc Am Soc Clin Oncol 5: 191, 1986 (Abstr)

17. Radice PA, Bunn Jr PA, Ihde DC: Therapeutic Trials with VP-16-213 and VM 26: Active Agents in Small Cell Lung Cancer, Non-Hodgkin's Lymphoma and Other Malignancies. Cancer Treat Rep 63:1231-39, 1979

18. Nissen NI, Pajak TF, Leone LA, Bloomfield CD, Kennedy BJ, Ellison RE, Silver RT, Weiss RB, Cuttner J, Falkson G, Kung F, Bergevin PR, Holland JF: Clinical Trial of VP/16-213 (NSC141540) I.V. Twice Weekly in Advanced Neoplastic Disease. A Study by the Cancer and Leukemia Group B. Cancer 45:232-35, 1980

19. Issel BF, Crooke ST: Etoposide (VP-16-213). Cancer Treat Rev 6:107-24, 1979

20. Freireich E, Frei III E, Karon M: Methylglyoxal Bis (guanylhydrazone): A New Active Agent Against Acute Myelocytic Leukemia. Cancer Chemother Rep 16:183-86, 1962

21. Bender RA, Anderson T, Fisher RI, Young RC: Activity of the Epipodophyllotoxin VP-16 in the Treatment of Combination Chemotherapy-Resistant Non-Hodgkin Lymphoma. Amer J Hematol 5:203-09, 1978

22. Knight III WAT, Fabian C, Constanzi JJ, Jones SE, Coltman Jr CA: Methyl-glyoxal Bis Guanylhydrazone (Methyl-gag, MGBG) in Lymphoma and Hodgkin's Disease. Invest New Drugs 1:235-7, 1983

23. Warrell RP, Lee BJ, Kempin SJ, Lacher MJ, Straus DJ, Young CW: Effectiveness of Methyl-Gag (MethylglyoxalBis [Guanylhydrazone]) in Patients with Advanced Malignant Lymphoma. Blood 57:1011-14, 1981

Address for offprints: Lyubica Dabich, 3119 Taubman Center, University of Michigan Medical Center, 1500 E. Medical Center Drive, Ann Arbor, Michigan 49109-0374, USA 